\title{
The center of a Leavitt path algebra
}

\section{Gonzalo Aranda Pino and Kathi Crow}

\begin{abstract}
In this paper the center of a Leavitt path algebra is computed for a wide range of situations. A basis as a $K$-vector space is found for $Z(L(E))$ when $L(E)$ enjoys some finiteness condition such as being artinian, semisimple, noetherian and locally noetherian. The main result of the paper states that a simple Leavitt path algebra $L(E)$ is central (i.e. the center reduces to the base field $K$ ) when $L(E)$ is unital and has zero center otherwise. Finally, this result is extended, under some mild conditions, to the case of exchange Leavitt path algebras.
\end{abstract}

\section{Introduction}

Leavitt path algebras of row-finite graphs have been recently introduced in [1] and [8]. They have become a subject of significant interest, both for algebraists and for analysts working in $C^{*}$-algebras. The Cuntz-Krieger algebras $C^{*}(E)$ (the $C^{*}$-algebra counterpart of these Leavitt path algebras, where $E$ denotes a graph) are described in [23]. The algebraic and analytic theories, while sharing some striking similarities, present some remarkable differences, as was shown for instance in the "Workshop on Graph Algebras" [14], and more deeply in the subsequent enlightening works [25, 7]. As noted in [7], the interplay between these two classes of graph algebras has been extensive and mutually beneficial. Graph $C^{*}$-algebra results have helped to guide the development of Leavitt path algebras by suggesting the veracity of some conjectures and by suggesting directions in which investigations should be focused. Similarly, Leavitt path algebras have given a better understanding of graph $C^{*}$-algebras by helping to identify those aspects of $C^{*}(E)$ that are algebraic in nature.

2000 Mathematics Subject Classification: 16D70.

Keywords: Leavitt path algebra, graph algebra, center. 
On the algebraic side of the picture, the algebras $L(E)$ are natural generalizations of the algebras investigated by Leavitt in [21], and are a specific type of path $K$-algebras ( $K$ is an arbitrary field) associated to a graph $E$ modulo certain relations. The family of algebras which can be realized as Leavitt path algebras of graphs includes matrix rings $\mathbb{M}_{n}(K)$ for $n \in \mathbb{N} \cup\{\infty\}$ (where $\mathbb{M}_{\infty}(K)$ denotes matrices of countably infinite size with only a finite number of nonzero entries), the Toeplitz algebra $T$, the Laurent polynomial ring $K\left[x, x^{-1}\right]$, and the classical Leavitt algebras $L(1, n)$ for $n \geq 2$. Constructions such as direct sums, direct limits, and matrices over the previous examples can be also realized in this setting. But, in addition to the fact that this class of algebras indeed includes many well-known algebras, one of the main interests in their study is the pictorial representations that their corresponding graphs provide.

A great deal of effort has been focused on trying to understand the algebraic structure of $L(E)$ via the graph nature of $E$. Concretely, the literature on Leavitt path algebras includes necessary and sufficient conditions on a graph $E$ so that the corresponding Leavitt path algebra $L(E)$ is simple [1], purely infinite simple [2], exchange [12], finite-dimensional [4], locally finite (equivalently noetherian) [5] and semisimple [6]. Another remarkable approach has been the research (explored quite intensively in [8], and touched on in [6]) of the monoids of finitely generated projective modules $V(L(E)$ ). Furthermore, several important substructures have been computed for these types of algebras, including: the socle [11], the Jacobson radical [3] and the left singular ideal [24], all of which have in turn become valuable tools in this theory.

However, the center of these Leavitt path algebras, $Z(L(E))$, seemed to have passed unnoticed and has never been explicitly computed. This is the main aim of this paper. Concretely, we compute the center of $L(E)$ under any of the properties described above, which can be grouped into essentially three categories: (1) $L(E)$ enjoys some finiteness condition, $(2) L(E)$ is simple, and (3) $L(E)$ is exchange.

This enterprise is a compulsory as well as a natural one. Although we clearly seek to benefit from the computation of $Z(L(E))$ from the algebraic point of view by having such a tool available, we also hope and foresee that the $C^{*}$-analytic side will be benefited from this work. In the theory of general $C^{*}$-algebras, the (extended) centroid is indeed a valuable tool as well (see for instance $[9])$.

This article is organized as follows. The Preliminaries section includes the basic definitions and examples that will be used throughout. In addition, we describe several graph constructions and more specific but general properties of $L(E)$ that will be of use in the rest of the paper. 
The first step of the investigation is carried out in Section 2, where a useful decomposition result about almost disjoint unions of graphs is proved and commutative Leavitt path algebras are analyzed.

The center is studied under finiteness conditions in Section 3. Specifically, the conditions of being artinian, semisimple, noetherian and locally noetherian are examined. The proofs there rely on the fact that for those conditions there exist structural theorems which allow one to conveniently trace back the elements of $L(E)$ so that, for each case, a $K$-basis of the center $Z(L(E))$ can be exhibited.

A completely different approach is taken in Section 4, where the main result of the paper, Theorem 4.2, is presented: a simple Leavitt path algebra $L(E)$ is either central (i.e. the center reduces to the base field $K$ ) when $L(E)$ is unital, or has zero center otherwise. The methods here differ from those in the previous section as there are no theorems characterizing simple Leavitt path algebras via isomorphisms to well-known families of algebras. However, these algebras do have a graph-theoretical structure theorem (namely, $L(E)$ is simple if and only if $E$ is cofinal and satisfies Condition (L) [1, Theorem 3.11]), which is heavily relied upon to establish Theorem 4.2.

The subsequent and final steps are pursued in Section 5, where the center for exchange Leavitt path algebras is considered. It is well known that simple Leavitt path algebras are all exchange, so studying the center for this broader family of Leavitt path algebras is a natural extension of the results from Section 4. Hence, under some mild hypotheses, it is shown that the center of an exchange Leavitt path algebra is isomorphic to $\bigoplus_{i=1}^{m} K$ for some $m \geq 0$. As with the simplicity result, a graph characterization of $E$ (the so-called Condition $(\mathrm{K})$ ) when $L(E)$ is exchange turns out to be key in the proofs.

\section{Preliminaries}

A (directed) graph $E=\left(E^{0}, E^{1}, r, s\right)$ consists of two countable sets $E^{0}, E^{1}$ and maps $r, s: E^{1} \rightarrow E^{0}$. The elements of $E^{0}$ are called vertices and the elements of $E^{1}$ are called edges. If $s^{-1}(v)$ is a finite set for every $v \in E^{0}$, then the graph is called row-finite. Throughout this paper we will be concerned only with row-finite graphs. If $E^{0}$ is finite, then, by the row-finiteness hypothesis, $E^{1}$ must necessarily be finite as well; in this case we simply say that $E$ is finite. A vertex which emits no edges is called a sink. A path $\mu$ in a graph $E$ is a sequence of edges $\mu=e_{1} \ldots e_{n}$ such that $r\left(e_{i}\right)=s\left(e_{i+1}\right)$ for $i=1, \ldots, n-1$. In this case, $s(\mu):=s\left(e_{1}\right)$ is the source of $\mu, r(\mu):=r\left(e_{n}\right)$ is the range of $\mu$, and $n$ is the length of $\mu$, which we denote by $l(\mu)$. For $n \geq 2$ we define $E^{n}$ to be the set of paths of length $n$, and $E^{*}=\bigcup_{n \geq 0} E^{n}$ the set of all paths. Throughout the paper $K$ will denote an arbitrary field. 
We define the Leavitt path $K$-algebra $L_{K}(E)$, or simply $L(E)$ if the base field is understood, as the $K$-algebra generated by a set $\left\{v \mid v \in E^{0}\right\}$ of pairwise orthogonal idempotents, together with a set of variables $\left\{e, e^{*} \mid e \in E^{1}\right\}$, which satisfy the following relations:

(1) $s(e) e=e r(e)=e$ for all $e \in E^{1}$.

(2) $r(e) e^{*}=e^{*} s(e)=e^{*}$ for all $e \in E^{1}$.

(3) $e^{*} e^{\prime}=\delta_{e, e^{\prime}} r(e)$ for all $e, e^{\prime} \in E^{1}$.

(4) $v=\sum_{\left\{e \in E^{1} \mid s(e)=v\right\}} e e^{*}$ for every $v \in E^{0}$ that emits edges.

Relations (3) and (4) are called the Cuntz-Krieger relations and are referred to as (CK1) and (CK2) respectively.

The elements of $E^{1}$ are called real edges, while for $e \in E^{1}$ we call $e^{*}$ a ghost edge. The set $\left\{e^{*} \mid e \in E^{1}\right\}$ will be denoted by $\left(E^{1}\right)^{*}$. We let $r\left(e^{*}\right)$ denote $s(e)$, and we let $s\left(e^{*}\right)$ denote $r(e)$. If $\mu=e_{1} \ldots e_{n}$ is a path, then we denote by $\mu^{*}$ the element $e_{n}^{*} \ldots e_{1}^{*}$ of $L(E)$, and by $\mu^{0}$ the set of its vertices, i.e., $\left\{s\left(e_{1}\right), r\left(e_{i}\right) \mid i=1, \ldots, n\right\}$. It was shown in [1, Lemma 1.5] that every monomial in $L(E)$ is either of the form $k v$, with $k \in K$ and $v \in E^{0}$, or of the form $k e_{1} \ldots e_{m} f_{1}^{*} \ldots f_{n}^{*}$ for $k \in K, m, n \in \mathbb{N}, e_{i}, f_{j} \in E^{1}$.

We call $\rho$ an undirected path in $E$ if there exist $\rho_{i} \in E^{1} \cup\left(E^{1}\right)^{*}$ so that $\rho=\rho_{1} \ldots \rho_{n}$ and $r\left(\rho_{i}\right)=s\left(\rho_{i+1}\right)$ for $1 \leq i \leq n-1$. We say that such a $\rho$ is an undirected path from a vertex $v$ to a vertex $w$ if $s\left(\rho_{1}\right)=v$ and $r\left(\rho_{n}\right)=w$. Note that we do not generally think of $\rho$ as an element of $L(E)$ as we are ignoring the CK-relations in this definition. We say that the graph $E$ is connected if given any two vertices, $v$ and $w$, there exists an undirected path from $v$ to $w$. For any subset $H$ of $E^{0}$, we will denote by $I(H)$ the ideal of $L(E)$ generated by $H$.

Note that if $E$ is a finite graph then we have $\sum_{v \in E^{0}} v=1_{L(E)}$. On the other hand, if $E^{0}$ is infinite, then by [1, Lemma 1.6] $L(E)$ is a nonunital ring with a set of local units. In fact, in this situation, $L(E)$ is a ring with enough idempotents (see e.g. [18] or [25]), and we have the decomposition $L(E)=\bigoplus_{v \in E^{0}} L(E) v$ as left $L(E)$-modules. (Equivalently, we have $L(E)=$ $\bigoplus_{v \in E^{0}} v L(E)$ as right $L(E)$-modules.)

Examples 1.1. By considering some basic configurations one can realize many algebras as the Leavitt path algebra of some graph. Here we give some well-known examples; the isomorphisms for the first three are given in [1], the fourth in [2], and the last in [24].

The ring of Laurent polynomials $K\left[x, x^{-1}\right]$ is the Leavitt path algebra of the following graph $R_{1}$ : 
Matrix algebras $M_{n}(K)$ can be achieved by considering a line graph with $n$ vertices and $n-1$ edges.

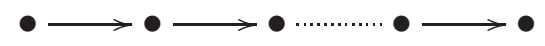

Classical Leavitt algebras $L(1, n)$ for $n \geq 2$ are obtained as $L\left(R_{n}\right)$ where $R_{n}$ is the rose with $n$ petals graph, shown below.

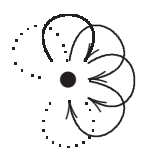

Of course, combinations of the previous examples are possible. For example, the Leavitt path algebra of the graph

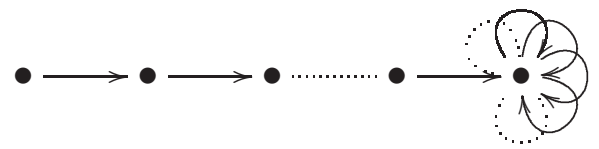

is $M_{n}(L(1, m))$, where $n$ denotes the number of vertices in the graph and $m$ denotes the number of loops. In addition, the algebraic counterpart of the Toeplitz algebra $T$ is the Leavitt path algebra of the graph $E$ having one loop and one exit.

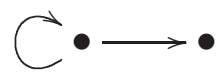

It is shown in [1] that $L(E)$ is a $\mathbb{Z}$-graded $K$-algebra, spanned as a $K$ vector space by $\left\{p q^{*} \mid p, q\right.$ are paths in $\left.E\right\}$. In particular, for each $n \in \mathbb{Z}$, the degree $n$ component $L(E)_{n}$ is spanned by elements of the form $p q^{*}$ where $l(p)-l(q)=n$. The degree of an element $x$, denoted $\operatorname{deg}(x)$, is the smallest number $n$ for which $x \in \bigoplus_{m<n} L(E)_{m}$.

We will analyze the structure of various graphs in the sequel. An important role is played by the following concepts. An edge $e$ is an exit for a path $\mu=e_{1} \ldots e_{n}$ if there exists $i$ such that $s(e)=s\left(e_{i}\right)$ and $e \neq e_{i}$. If $\mu$ is a path in $E$ with $v=s(\mu)=r(\mu)$ then $\mu$ is a closed path based at $v$. If $\mu$ is a closed path based at $v$ such that $s\left(e_{i}\right) \neq v$ for every $i>1$, then $\mu$ is a closed simple path based at $v$. If $s(\mu)=r(\mu)$ and $s\left(e_{i}\right) \neq s\left(e_{j}\right)$ for every $i \neq j$, then $\mu$ is called a cycle. A graph which contains no cycles is called acyclic. We say that a graph $E$ satisfies Condition (L) if every cycle in $E$ has an exit. Following [20], a graph $E$ satisfies Condition $(\mathrm{K})$ if for each vertex $v$ on a closed simple path there exist at least two distinct closed simple paths $\alpha, \beta$ based at $v$.

We define a relation $\geq$ on $E^{0}$ by setting $v \geq w$ if there is a path $\mu$ in $E$ with $s(\mu)=v$ and $r(\mu)=w$. A subset $H$ of $E^{0}$ is called hereditary if $v \geq w$ and $v \in H$ imply $w \in H$. A hereditary set is saturated if every vertex 
which feeds into $H$ and only into $H$ is itself in $H$, that is, if $s^{-1}(v) \neq \emptyset$ and $r\left(s^{-1}(v)\right) \subseteq H$ imply $v \in H$. Denote by $\mathcal{H}_{E}$ the set of hereditary saturated subsets of $E^{0}$. A graph $E$ is cofinal if $\mathcal{H}_{E}=\left\{\emptyset, E^{0}\right\}$ (see for instance [12, Lemma 2.8]).

The set $T(v)=\left\{w \in E^{0} \mid v \geq w\right\}$ is the tree of $v$, and it is the smallest hereditary subset of $E^{0}$ containing $v$. We extend this definition for an arbitrary set $X \subseteq E^{0}$ by $T(X)=\bigcup_{x \in X} T(x)$. The hereditary saturated closure of a set $X$ is defined as the smallest hereditary and saturated subset of $E^{0}$ containing $X$. It is shown in $[8,15]$ that the hereditary saturated closure of a set $X$ is $\bar{X}=\bigcup_{n=0}^{\infty} \Lambda_{n}(X)$, where

$$
\begin{aligned}
& \Lambda_{0}(X)=T(X), \quad \text { and, for } n \geq 1 \\
& \Lambda_{n}(X)=\left\{y \in E^{0} \mid s^{-1}(y) \neq \emptyset \text { and } r\left(s^{-1}(y)\right) \subseteq \Lambda_{n-1}(X)\right\} \cup \Lambda_{n-1}(X)
\end{aligned}
$$

Recall that an ideal $J$ of $L(E)$ is graded if and only if it is generated by idempotents; in fact, $J=I(H)$, where $H=J \cap E^{0} \in \mathcal{H}_{E}$. (See the proofs of [8, Proposition 4.2 and Theorem 4.3].) We will use this fact freely throughout.

We recall here some graph-theoretic constructions which will be of interest. For a hereditary subset $H$ of $E^{0}$, the quotient graph $E / H$ is defined as

$$
\left(E^{0} \backslash H,\left\{e \in E^{1} \mid r(e) \notin H\right\},\left.r\right|_{(E / H)^{1}},\left.s\right|_{(E / H)^{1}}\right) .
$$

The following construction was originally introduced in [16, Definition 1.3]: let $E$ be a graph, and let $\emptyset \neq H \in \mathcal{H}_{E}$. Define

$$
F_{E}(H)=\left\{\alpha_{1} \ldots \alpha_{n} \mid \alpha_{i} \in E^{1}, s\left(\alpha_{1}\right) \notin H, r\left(\alpha_{i}\right) \notin H \text { for } i<n, r\left(\alpha_{n}\right) \in H\right\} .
$$

Denote by $\bar{F}_{E}(H)$ another copy of $F_{E}(H)$. For $\alpha \in F_{E}(H)$, we write $\bar{\alpha}$ to denote a copy of $\alpha$ in $\bar{F}_{E}(H)$. Then, we define the graph

$$
{ }_{H} E=\left({ }_{H} E^{0},{ }_{H} E^{1}, s^{\prime}, r^{\prime}\right)
$$

as follows:

1. $\left({ }_{H} E\right)^{0}=H \cup F_{E}(H)$.

2. $\left({ }_{H} E\right)^{1}=\left\{e \in E^{1} \mid s(e) \in H\right\} \cup \bar{F}_{E}(H)$.

3. For every $e \in E^{1}$ with $s(e) \in H, s^{\prime}(e)=s(e)$ and $r^{\prime}(e)=r(e)$.

4. For every $\bar{\alpha} \in \bar{F}_{E}(H), s^{\prime}(\bar{\alpha})=\alpha$ and $r^{\prime}(\bar{\alpha})=r(\alpha)$. 


\section{Decomposable and commutative Leavitt path alge- bras}

In this section we give a decomposition result for Leavitt path algebras that generalizes the one of [4]. Specifically, we prove that for certain nice families of subgraphs $\left\{E_{i}\right\}_{i \in I}$ of a graph $E$ we have a direct sum decomposition $L(E) \cong \bigoplus_{i \in I} L\left(E_{i}\right)$. The reason for seeking such a result is twofold. First, it will allow us to establish the commutativity result of this section; second, it is useful as a further step in computing the center of the Leavitt path algebras because if we know the center of the Leavitt path algebras of these subgraphs (because $L\left(E_{i}\right)$ are simple, say), then we can recover the center of $L(E)$ via the property $Z\left(\bigoplus_{i \in I} R_{i}\right)=\bigoplus_{i \in I} Z\left(R_{i}\right)$ for rings $R_{i}$, a property which we will often use without explicit mention.

Definition 2.1. Let $E$ be a graph. Given a collection $\left\{E_{i}\right\}_{i \in I}$ of subgraphs of $E$, we say that $E$ is an almost disjoint union of $\left\{E_{i}\right\}_{i \in I}$ if the following conditions are satisfied:

(i) $E^{0}=\bigcup_{i \in I} E_{i}^{0}$.

(ii) $r_{E}^{-1}\left(E_{i}^{0} \cap E_{j}^{0}\right)=\emptyset$ for every $i \neq j$.

(iii) $E_{i}^{0} \cap E_{j}^{0} \subseteq s_{E}\left(E_{i}^{1}\right) \cap s_{E}\left(E_{j}^{1}\right)$ for every $i \neq j$.

(iv) $\left\{E_{i}^{1}\right\}_{i \in I}$ is a partition of $E^{1}$.

In this situation we will write $E=\coprod_{i \in I} E_{i}$.

Remark 2.2. Even though the subgraphs $E_{i}$ in Definition 2.1 do not have common edges, they might have common vertices, as in the following example. Of course, if the given subgraphs do not have common vertices then the almost disjoint union is a disjoint union of subgraphs. Note that condition (ii) means that any two distinct subgraphs have either no common vertices, or they have common vertices which were sources in $E$. In addition, condition (iii) can be interpreted as follows: whenever we have a common vertex $v \in E_{i}^{0} \cap E_{j}^{0}$, then it emits some edge $e$ in $E_{i}^{1}$ as well as some edge $f$ in $E_{j}^{1}$.

Example 2.3. If $E, E_{1}$, and $E_{2}$ are as below then $E=E_{1} \coprod E_{2}$.

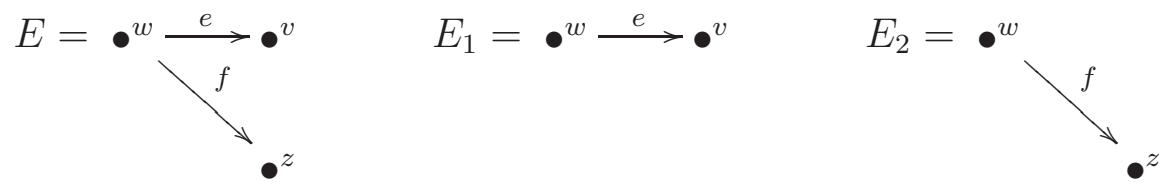


Proposition 2.4. Suppose that a graph $E$ can be written as the almost disjoint union of subgraphs $E_{i}$, that is, $E=\coprod_{i \in I} E_{i}$, where $|I| \leq \aleph_{0}$. Then $L(E) \cong \bigoplus_{i \in I} L\left(E_{i}\right)$.

Proof. For each $v \in E^{0}$, write $J_{v}=\left\{j \in I \mid v \in E_{j}^{0}\right\}$ and for any $J \subseteq I$, define $\delta_{i J}$ as 1 whenever $i \in J$ and zero otherwise. We will produce a map $\varphi: L(E) \rightarrow \bigoplus_{i \in I} L\left(E_{i}\right)$ by defining it on the generators of $L(E)$ and extending linearly and multiplicatively as follows. Define $\varphi(v):=\left(\delta_{i J_{v}} v\right)_{i \in I}$. Note that we have an element of the direct sum $\bigoplus_{i \in I} L\left(E_{i}\right)$ precisely because of conditions (iii) and (iv) of Definition 2.1 and the fact that the graph $E$ is row-finite.

For a real edge $e \in E^{1}$ (analogously for a ghost edge $e^{*} \in\left(E^{1}\right)^{*}$ ) we define $\varphi(e):=\left(\delta_{i j} e\right)_{i \in I}$, where $j$ is the unique index in $I$ such that $e \in E_{j}^{1}$, which exists by condition (iv).

We extend linearly and multiplicatively to all of $L(E)$. This clearly gives us an algebra homomorphism once we prove that $\varphi$ is well-defined, that is, it preserves the relations defining $L(E)$. This is a straightforward computation done by cases; we present a few sample cases here.

For the relation $s(e) e=e$ we let $j \in I$ be the unique index such that $e \in E_{j}^{1}$. Then the definition of $\varphi$ gives us

$$
\varphi(s(e) e)=\left(\delta_{i J_{s(e)}} s(e)\right)_{i \in I}\left(\delta_{i j} e\right)_{i \in I}=\left(\delta_{i j} s(e) e\right)_{i \in I}=\left(\delta_{i j} e\right)_{i \in I}=\varphi(e)
$$

where we are making obvious uses of the facts that $j \in J_{s(e)}$ (because $E_{j}$ is a subgraph of $E$ ) and that the relation $s(e) e=e$ is satisfied in $E_{j}$.

Let us focus now on the relation $e^{*} e=r(e)$. Clearly enough, $r(e)$ is not a source so that by condition (ii) of Definition 2.1 we get that $r(e)$ is in only one subgraph $E_{j}$ (that is, $J_{r(e)}=\{j\}$ ). This subgraph must coincide with the unique subgraph containing both $e$ and $e^{*}$. Therefore, by using the relation $e^{*} e=r(e)$ in $E_{j}$ we get that

$$
\begin{aligned}
\varphi\left(e^{*} e\right) & =\varphi\left(e^{*}\right) \varphi(e)=\left(\delta_{i j} e^{*}\right)_{i \in I}\left(\delta_{i j} e\right)_{i \in I}=\left(\delta_{i j} e^{*} e\right)_{i \in I}=\left(\delta_{i j} r(e)\right)_{i \in I} \\
& =\left(\delta_{i J_{r(e)}} r(e)\right)_{i \in I}=\varphi(r(e)) .
\end{aligned}
$$

Finally, let us consider the (CK2) relation in $E^{0}, v=\sum_{\left\{e \in E^{1} \mid s(e)=v\right\}} e e^{*}$. By the row-finiteness condition we can rename as $e_{1}, \ldots, e_{m}$ the edges in the previous summation and by condition (iv) we may rearrange them to find $j_{0}=0 \leq j_{1} \leq \cdots \leq j_{n}=m$ and $i_{1}, \ldots, i_{n} \in I$ such that $e_{j_{k-1}+1}, \ldots, e_{j_{k}} \in$ $E_{i_{k}}^{1}$ for every $k=1, \ldots, n$. Now condition (iii) ensures that $J_{v}=\left\{i_{1}, \ldots, i_{n}\right\}$. Moreover, condition (iv) allows us to obtain (CK2) relations in each $E_{i_{k}}$ as 
$v=e_{j_{k-1}+1} e_{j_{k-1}+1}^{*}+\cdots+e_{j_{k}} e_{j_{k}}^{*}$. Putting all this information together we have

$$
\begin{aligned}
\varphi\left(\sum_{\left\{e \in E^{1} \mid s(e)=v\right\}} e e^{*}\right) & =\sum_{k=1}^{n} \sum_{l=j_{k-1}+1}^{j_{k}} \varphi\left(e_{l}\right) \varphi\left(e_{l}^{*}\right)=\sum_{k=1}^{n} \sum_{l=j_{k-1}+1}^{j_{k}}\left(\delta_{i i_{k}} e_{l} e_{l}^{*}\right)_{i \in I} \\
& =\sum_{k=1}^{n}\left(\delta_{i i_{k}} v\right)_{i \in I}=\left(\delta_{i J_{v}} v\right)_{i \in I}=\varphi(v) .
\end{aligned}
$$

This proves that $\varphi$ is a well-defined $K$-algebra homomorphism. Clearly $\varphi(v) \neq 0$ for every $v \in E^{0}$ by condition (i) of Definition 2.1, and by endowing $\bigoplus_{i \in I} L\left(E_{i}\right)$ with the natural $\mathbb{Z}$-grading inherited by its components, we have that $\varphi$ is a $\mathbb{Z}$-graded algebra homomorphism. Therefore, the Graded Uniqueness Theorem [25, Theorem 4.8] yields the injectivity of $\varphi$.

In order to check that $\varphi$ is surjective, it is enough to show that, for each $j \in I$ and any element $x \in E_{j}^{0} \cup E_{j}^{1} \cup\left(E_{j}^{1}\right)^{*}$, we have $\left(\delta_{i j} x\right)_{i \in I} \in \operatorname{Im}(\varphi)$. This is clear for the edges and the ghost edges. Fix $j \in I$ and pick $v \in E_{j}^{0}$. We distinguish two situations. First, if $v$ is a sink in $E_{j}$ then, by condition (iii), $v \notin E_{k}^{0}$ for any $k \neq j$, so that $J_{v}=\{j\}$ and therefore $\varphi(v)=\left(\delta_{i J_{v}} v\right)_{i \in I}=$ $\left(\delta_{i j} v\right)_{i \in I}$. Second, if $v$ is not a sink in $E_{j}$, then we have a (CK2) relation at $v$ in $E_{j}$, which we can write as $v=\sum_{\left\{e \in E_{j}^{1} \mid s(e)=v\right\}} e e^{*}$ and therefore

$$
\varphi\left(\sum_{\left\{e \in E_{j}^{1} \mid s(e)=v\right\}} e e^{*}\right)=\left(\delta_{i j} \sum_{\left\{e \in E_{j}^{1} \mid s(e)=v\right\}} e e^{*}\right)_{i \in I}=\left(\delta_{i j} v\right)_{i \in I},
$$

and the proof is complete.

Remark 2.5. The conclusion of Proposition 2.4 might fail if any of the conditions of Definition 2.1 are not satisfied. There are trivial counterexamples for conditions (i) and (iv), as they essentially "gather" the graph. For condition (ii) we consider the graph $E$ and its two subgraphs $E_{1}$ and $E_{2}$ given by the following pictures.

$$
E=e C \bullet^{v} \stackrel{f}{\longrightarrow} \bullet^{w} \quad E_{1}=e \bullet^{v} \quad E_{2}=\bullet^{v} \stackrel{f}{\longrightarrow} \bullet^{w}
$$

This decomposition of $E$ into these two subgraphs satisfies all the conditions for being an almost disjoint union with the exception of condition (ii). Thus one might expect that $L(E) \cong L\left(E_{1}\right) \oplus L\left(E_{2}\right)$, but $L(E)$ is isomorphic to the Toeplitz algebra (see [24, Definition 5.1]) and hence is prime by [13, Corollary 3.10] whereas $L\left(E_{1}\right) \oplus L\left(E_{2}\right)$ obviously is not. For condition (iii) consider the following graph $E$ and its two subgraphs $E_{1}$ and $E_{2}$.

$$
E=\bullet \stackrel{f}{\longrightarrow} \bullet w \quad E_{1}=\bullet v \quad E_{2}=\bullet \stackrel{f}{\longrightarrow} \bullet w
$$


For this decomposition, every condition in Definition 2.1 is satisfied except for condition (iii). Applying [4, Proposition 3.5] we obtain

$$
L(E) \cong \mathbb{M}_{2}(K) \neq K \oplus \mathbb{M}_{2}(K) \cong L\left(E_{1}\right) \oplus L\left(E_{2}\right) .
$$

Remark 2.6. As noted before, some direct sum decomposition results were given in [4, Lemmas 4.4 and 5.4]. However, those are restricted to certain arrangements of finite and acyclic graphs, so that one always obtains direct sums of finite matrix algebras. In fact, their proofs rely solely on the structure result for Leavitt path algebras of finite and acyclic graphs [4, Proposition 3.5], so these results do not recover the structure of $L(E)$ via that of the $L\left(E_{i}\right)$, as in Proposition 2.4, but rather they treat $L(E)$ as a whole and note that it turns out to be a direct sum of matrices. Moreover, both operations $\bigwedge_{i \in I} E_{i}$ and $\bigvee_{i \in I} E_{i}$ used in [4] are particular cases of $\coprod_{i \in I} E_{i}$.

We are now in a position to completely determine the commutative Leavitt path algebras.

Proposition 2.7. Let $E$ be a graph. The following conditions are equivalent.

(i) $L(E)$ is commutative.

(ii) $E$ is such that the maps $r$ and $s$ coincide and are injective.

(iii) $E=\coprod_{i \in I} E_{i}$, where $|I| \leq \aleph_{0}$ and each subgraph $E_{i}$ is either an isolated vertex or the graph $R_{1}$ (an isolated loop).

(iv) $L(E) \cong \bigoplus_{i \in I_{1}} K \oplus \bigoplus_{i \in I_{2}} K\left[x, x^{-1}\right]$, where $\left|I_{1}\right|,\left|I_{2}\right| \leq \aleph_{0}$.

Proof. (i) $\Rightarrow$ (ii). Suppose that there exists $e \in E^{1}$ with $s(e) \neq r(e)$. In this situation we have $r(e)=e^{*} e=e e^{*}$ by commutativity. Now, $r(e)=$ $r(e)^{2}=r(e)\left(e e^{*}\right)=r(e) s(e)\left(e e^{*}\right)=0$, which is absurd as vertices are always nonzero elements of $L(E)$ (this fact has been noted in many papers, but it was only recently proved in [19, Lemma 1.5]). This shows that $s=r$.

Let us consider $e, f \in E^{1}$ with $s(e)=s(f)$. Suppose that $e \neq f$. By the commutativity of $L(E)$ we get $f=s(f) f=s(e) f=r(e) f=e^{*} e f=e^{*} f e=$ $0 e=0$, which would imply that $r(f)=f^{*} f=f^{*} 0=0$, a contradiction to [19, Lemma 1.5] again.

(ii) $\Rightarrow$ (iii) is straightforward.

(iii) $\Rightarrow$ (iv). Apply Proposition 2.4 and the well-known facts that $L\left(R_{1}\right) \cong$ $K\left[x, x^{-1}\right]$ and $L(\{v\}) \cong K$.

(iv) $\Rightarrow$ (i) is evident. 


\section{The center of Leavitt path algebras with finiteness conditions}

There are situations when the structure of Leavitt path algebras can be completely determined. This happens when $L(E)$ has any of these finiteness conditions: artinian, semisimple, noetherian or locally noetherian. In these cases we will be able to determine the center of $L(E)$ precisely because $L(E)$ turns out to be isomorphic to some well-known algebra (these are certain products of adequate matrices over the field $K$ or over Laurent polynomial algebras $\left.K\left[x, x^{-1}\right]\right)$. Furthermore, not only can we determine the structure of $L(E)$, but we can also give an explicit algebra isomorphism. This correspondence allows us to find a basis for the center of these families of algebras. The main results of this section are the computations of these centers.

The result which follows is well known for rings with identity. We provide here a proof for rings with local units (Leavitt path algebras might not be unital, but they are rings with local units).

For us, by a countable set we mean a set which is either finite or countably infinite. The symbol $\mathbb{M}_{\infty}(K)$ will denote the $K$-algebra of matrices over $K$ of countable size but with only a finite number of nonzero entries.

Lemma 3.1. Let $R$ be a ring with local units and $n \in \mathbb{N}$. Then

$$
Z\left(\mathbb{M}_{n}(R)\right)=Z(R) \operatorname{Id}_{n}=\left\{\left(\begin{array}{cccc}
a & 0 & \ldots & 0 \\
0 & a & \ldots . & 0 \\
\vdots & \ddots & \\
0 & 0 & \ldots & a
\end{array}\right) \mid a \in Z(R)\right\} \cong Z(R)
$$

and $Z\left(\mathbb{M}_{\infty}(R)\right)=0$.

Proof. Suppose $m \in \mathbb{N} \cup\{\infty\}$ and consider a matrix $\left(a_{k l}\right) \in Z\left(\mathbb{M}_{m}(R)\right)$. Let $i \neq j$ and let $e \in R$ be a local unit for the entry $a_{j i} \in R$. For an element $a \in R$, denote by $a[i j]$ the matrix which has $a$ in the $(i, j)$-entry and zero elsewhere. Then, $e[i j]\left(a_{k l}\right) e[i j]=\left(e a_{j i} e\right)[i j]=\left(a_{j i}\right)[i j]$, but $\left(a_{k l}\right)$ is in the center, so that $e[i j]\left(a_{k l}\right) e[i j]=\left(a_{k l}\right) e[i j] e[i j]=\left(a_{k l}\right) 0=0$. Therefore $a_{i j}=0$. Let $f \in R$ be a local unit for both $a_{i i}$ and $a_{j j}$. Now, $f[i j]\left(a_{k l}\right)=$ $\left(f a_{j j}\right)[i j]=\left(a_{j j}\right)[i j]=\left(a_{k l}\right) f[i j]=\left(a_{i i} f\right)[i j]=\left(a_{i i}\right)[i j]$, so that $a_{i i}=a_{j j}$. In the case of $m=\infty$, only finitely many entries of $\left(a_{k l}\right)$ are nonzero so $a_{i i}=0$ and hence $\left(a_{k l}\right)=0$. The converse containment is obvious and the result follows.

The first situation where we have finiteness conditions in Leavitt path algebras is when these are finite-dimensional and, as we will see in the next proposition, for Leavitt path algebras this is equivalent to being one-sided artinian (and hence unital). The center of $L(E)$ under these hypotheses is computed in the next result. 
Proposition 3.2. If $L(E)$ is one-sided artinian then the following hold.

(i) $Z(L(E)) \cong \bigoplus_{i=1}^{n} K$, where $n$ is the number of sinks in $E$.

(ii) A basis of $Z(L(E))$ as a $K$-vector space is

$$
\left\{\sum_{k=1}^{m_{i}} p_{k, i} p_{k, i}^{*} \mid i=1, \ldots, n\right\}
$$

where $\left\{w_{1}, \ldots, w_{n}\right\}$ are the sinks and $\left\{p_{k, i} \mid k=1, \ldots, m_{i}\right\}$ are the paths ending in $w_{i}$.

Proof. If $L(E)$ is left (or right) artinian, in particular it is locally left (resp. right) artinian (see [17]) so that [6, Theorem 2.4] applies to yield that $L(E) \cong$ $\bigoplus_{i \in \Upsilon} \mathbb{M}_{m_{i}}(K)$, where $\Upsilon$ is a countable set and $m_{i} \in \mathbb{N} \cup\{\infty\}$. Clearly, the artinian condition implies in this case that both the direct sum and the sizes of the matrices are finite, so that $L(E)$ is finite dimensional. Applying [4, Corollary 3.6 and Proposition 3.5] yields that $L(E) \cong \bigoplus_{i=1}^{n} \mathbb{M}_{m_{i}}(K)$, where $n$ is the number of sinks of $E$. Now Lemma 3.1 proves (i) in the statement.

Moreover, the isomorphism $\varphi: L(E) \rightarrow \bigoplus_{i=1}^{n} \mathbb{M}_{m_{i}}(K)$ can be recovered by following the proofs of [5, Lemma 3.4 and Proposition 3.5]. Concretely: if $\left\{w_{1}, \ldots, w_{n}\right\}$ are the sinks in $E$ and $\left\{p_{k, i} \mid k=1, \ldots, m_{i}\right\}$ are the paths ending in $w_{i}$ as in (ii), then $\varphi^{-1}\left(\left(a_{k j}^{i}\right)_{i=1}^{n}\right)=\sum_{i=1}^{n} \sum_{k, j=1}^{m_{i}} p_{k, i} p_{j, i}^{*}$. Again an application of Lemma 3.1 gives (ii).

Using the results stated and the ideas contained in Lemma 3.1 and Proposition 3.2 we can determine the center of a semisimple Leavitt path algebra.

Corollary 3.3. If $L(E)$ is semisimple then the following hold.

(i) If $\left\{w_{i}\right\}_{i \in I}$ are the sinks in E which lie on only a finite number of paths, then

$$
Z(L(E)) \cong \bigoplus_{i \in I} K
$$

(ii) A basis of $Z(L(E))$ as a $K$-vector space is

$$
\left\{\sum_{k=1}^{m_{i}} p_{k, i} p_{k, i}^{*} \mid i \in I\right\}
$$

where $\left\{p_{k, i} \mid k=1, \ldots, m_{i}\right\}$ are the paths ending in $w_{i}$ for each $i \in I$.

The conditions of being left (or right) noetherian and locally left (or right) noetherian are handled in a fashion similar to the artinian situation, as shown below. 
Proposition 3.4. If $L(E)$ is one-sided noetherian then the following hold.

(i) $Z(L(E)) \cong\left(\bigoplus_{i=1}^{l} K\left[x, x^{-1}\right]\right) \oplus\left(\bigoplus_{j=1}^{l^{\prime}} K\right)$, where $l$ is the number of cycles in $E$ and $l^{\prime}$ is the number of sinks in $E$.

(ii) A basis of $Z(L(E))$ as a $K$-vector space is

$$
\left\{\sum_{k=1}^{m_{i}} p_{k, i} c_{i}^{r} p_{k, i}^{*} \mid r \in \mathbb{Z}, i=1, \ldots, l\right\} \cup\left\{\sum_{k=1}^{m_{i}} p_{k, i} p_{k, i}^{*} \mid i=l+1, \ldots, l+l^{\prime}\right\}
$$

where $c_{1}, \ldots, c_{l}$ are the cycles in $E$ and for each $1 \leq i \leq l,\left\{p_{k, i} \mid k=\right.$ $\left.1, \ldots, m_{i}\right\}$ are the paths ending in a fixed (although arbitrary) vertex $v_{i}$ of the cycle $c_{i}$ which do not contain the cycle itself; $v_{l+1}, \ldots, v_{l+l^{\prime}}$ are the sinks and for every $l+1 \leq i \leq l+l^{\prime},\left\{p_{k, i} \mid k=1, \ldots, m_{i}\right\}$ are the paths ending in the sink $v_{i}$.

Proof. First we use [5, Theorem 3.10] to get that $L(E)$ is a locally finite algebra (that is, its homogeneous components $L(E)_{n}$ for $n \in \mathbb{Z}$ are finite dimensional). In this situation [5, Theorem 3.8] applies to yield that

$$
L(E) \cong\left(\bigoplus_{i=1}^{l} \mathbb{M}_{m_{i}}\left(K\left[x, x^{-1}\right]\right)\right) \oplus\left(\bigoplus_{i=l+1}^{l+l^{\prime}} \mathbb{M}_{m_{i}}(K)\right)
$$

where $l, l^{\prime}$ and $m_{i}$ for $i=1, \ldots, l+l^{\prime}$ are as in (i) and (ii) of the statement.

Now, an application of Lemma 3.1 gives (i), and by following similar reasonings and proofs to that of Proposition 3.2 and [5, Theorem 3.8] we can trace back the elements in the diagonals of the matrices to obtain the basis for $Z(L(E))$ shown in (ii).

Finally, and paralleling what was done in the artinian case, we can generalize the previous result to the nonunital case, that is, for locally left (or right) noetherian: recall that a ring $R$ is locally left noetherian $[6$, Definition 1.4] if for any finite subset $S$ of $R$ there exists $e=e^{2} \in R$ such that $S \subseteq e R e$, with $e$ Re left (resp. right) noetherian.

To state the corollary, we need the following two definitions. A cycle $c$ is a finite path cycle if there are only a finite number of paths not containing $c$ which end at any vertex of $c$. A sink $w$ is a finite path sink if $w$ lies on only a finite number of paths.

Corollary 3.5. If $L(E)$ is locally one-sided noetherian then the following hold.

(i) If $\left\{c_{i}\right\}_{i \in I}$ is the set of finite path cycles in $E$ and $\left\{w_{j}\right\}_{j \in J}$ is the set of finite path sinks in $E$, then $Z(L(E)) \cong\left(\bigoplus_{i \in I} K\left[x, x^{-1}\right]\right) \oplus\left(\bigoplus_{j \in J} K\right)$. 
(ii) A basis of $Z(L(E))$ as a $K$-vector space is

$$
\left\{\sum_{k=1}^{m_{i}} p_{k, i} c_{i}^{r} p_{k, i}^{*} \mid r \in \mathbb{Z}, i \in I\right\} \cup\left\{\sum_{k=1}^{m_{j}} p_{k, j} p_{k, j}^{*} \mid j \in J\right\}
$$

where for each $i \in I,\left\{p_{k, i} \mid k=1, \ldots, m_{i}\right\}$ are the paths ending in a fixed (although arbitrary) vertex $v_{i}$ of the cycle $c_{i}$ which do not contain the cycle itself; and for every $j \in J,\left\{p_{k, j} \mid k=1, \ldots, m_{j}\right\}$ are the paths ending in the sink $w_{j}$.

Proof. Apply [6, Theorem 3.7 and its proof], Prop. 3.4 and Lemma 3.1.

\section{The center of simple Leavitt path algebras}

In the previous sections we bypassed the problem of calculating the centers of Leavitt path algebras by setting up isomorphisms between $L(E)$ and algebras whose centers are well-known. Here we will work directly with $L(E)$ and calculate the center of a simple Leavitt path algebra.

Before restricting our attention to simple Leavitt path algebras, we prove the following lemma about an element of the center of a Leavitt path algebra.

Lemma 4.1. Given a connected graph $E$, if $x=\sum_{v \in E^{0}} \lambda_{v} v \in Z(L(E))$ with $\lambda_{v} \in K$, then $\lambda_{v}=\lambda_{w}$ for all $v, w \in E^{0}$.

Proof. Suppose $e \in E^{1}$ with $s(e)=v$ and $r(e)=w$. Then we have $\lambda_{w} e=e x=x e=\lambda_{v} e$ and hence $\lambda_{v}=\lambda_{w}$, as the elements in $E^{1}$ are nonzero when considered as elements in $L(E)$ (for a proof of this fact see [24, Lemma 1.1] and [19, Lemma 1.5], where it is shown that they are linearly independent).

Proceeding by induction, assume that whenever there exists an undirected path of length $n$ between two vertices then the corresponding coefficients are equal. Now suppose there is an undirected path of length $n+1$ between two vertices, $v$ and $w$. Then there is a vertex $z$ with an undirected path of length $n$ between $v$ and $z$ and an edge $e$ between $z$ and $w$. Thus we have $\lambda_{v}=\lambda_{z}$ and $\lambda_{z}=\lambda_{w}$ and hence $\lambda_{v}=\lambda_{w}$.

We present here the main result of the paper where two things are shown. First, we prove that simple unital Leavitt path algebras are central, i.e. their centers reduce to the base field. But we also show that, unlike for the not necessarily simple case (see Proposition 2.7), nonunital simple Leavitt path algebras have zero center.

Theorem 4.2. If $L(E)$ is a simple algebra then $Z=Z(L(E))=K \cdot 1_{L(E)}$ if $L(E)$ is unital and $Z=0$ otherwise. 
Proof. Because $L(E)$ is a simple algebra, we know from [1, Theorem 3.11] that $E$ satisfies Condition (L) (i.e. that every cycle of $E$ has an exit) and that the only saturated and hereditary subsets of $E^{0}$ are the trivial ones. We will use these facts without further mention.

Suppose $x$ is a nonzero element of $Z$. Applying [11, Proposition 3.1], we obtain monomials $\alpha$ and $\beta$ and a nonzero element $k \in K$ so that $\alpha x \beta=k v$. Since $x \in Z$, we have that $\alpha x \beta=\alpha \beta x$. Now $\alpha \beta$ is a monomial and hence, as noted in Section 1, there exist paths $p$ and $q$ so that $\alpha \beta=p q^{*}$. Because $p q^{*} x=p x q^{*}=k v=k v^{2}$, we have that $p x q^{*} v=k v \neq 0$ and hence $q^{*} v \neq 0$. Therefore we must have $s(q)=r\left(q^{*}\right)=v$, so

$$
r(p) r(q) x=p^{*} p q^{*} q x=p^{*}\left(p q^{*} x\right) q=p^{*} k v q=k p^{*} q .
$$

Since $p q^{*} \neq 0$, we have $r(p)=r(q)$. Also, $p r(p) x q^{*}=k v \neq 0$ whence $r(p) x \neq 0$ and thus $p^{*} q \neq 0$. Therefore we have three options for $p^{*} q$, namely $p^{*} q \in E^{0}, p^{*} q$ is a closed (real) path, or $p^{*} q$ is a closed ghost path.

Let $\Theta_{0}=\left\{w \in E^{0} \mid w \leq r(p)\right\}$. For $w \in \Theta_{0}$, there is a path $\mu$ from $r(p)$ to $w$, so we have

$$
w x=\mu^{*} r(p) \mu x=k \mu^{*} p^{*} q \mu .
$$

Therefore we have that $w x$ must be $0, k$ times the vertex $w, k$ times a closed path, or $k$ times a closed ghost path and we can partition $\Theta_{0}$ into the following sets.

$$
\begin{aligned}
& \mathcal{V}=\left\{w \in \Theta_{0} \mid w x=k w\right\} \\
& \mathcal{P}=\left\{w \in \Theta_{0} \mid w x \text { is } k \text { times a closed real path }\right\} \\
& \mathcal{G}=\left\{w \in \Theta_{0} \mid w x \text { is } k \text { times a closed ghost path }\right\} \\
& \mathcal{N}=\left\{w \in \Theta_{0} \mid w x=0\right\}
\end{aligned}
$$

We will show that $\mathcal{P}, \mathcal{G}$, and $\mathcal{N}$ are empty so that $\Theta_{0}=\mathcal{V}$. First, we claim that the set $\hat{\mathcal{N}}=\left\{w \in E^{0} \mid w x=0\right\}$ is a hereditary and saturated subset of $E^{0}$ and hence is empty. To show this, suppose that $w \in \hat{\mathcal{N}}$ and $z \leq w$. Then there is a path $\rho$ with $s(\rho)=w$ and $r(\rho)=z$. Hence we have

$$
z x=\rho^{*} \rho x=\rho^{*} w x \rho=0 .
$$

Thus $z \in \hat{\mathcal{N}}$, so $\hat{\mathcal{N}}$ is hereditary. Now suppose that $w \in E^{0}$ so that $r\left(s^{-1}(w)\right)$ is a nonempty subset of $\hat{\mathcal{N}}$. Since $w=\sum_{e \in s^{-1}(w)} e e^{*}$, we have

$$
w x=\sum_{e \in s^{-1}(w)} e x e^{*}=\sum_{e \in s^{-1}(w)} e r(e) x e^{*}=0 .
$$

Therefore $w \in \hat{\mathcal{N}}$ and hence $\hat{\mathcal{N}}$ is a hereditary and saturated subset of $E^{0}$. Since $E^{0}$ has no proper hereditary and saturated subsets and $r(p) \notin \hat{\mathcal{N}}$, we have that $\hat{\mathcal{N}}=\emptyset$. As $\mathcal{N} \subseteq \hat{\mathcal{N}}$, it must be that $\mathcal{N}=\emptyset$ and thus $w x$ is nonzero for every vertex $w \in \Theta_{0}$. 
Next, suppose that $\mathcal{P} \neq \emptyset$. Then there is a vertex $w$ so that $w x=k c$, for some closed path $c$. Since $E$ satisfies Condition (L), every cycle has an exit and thus $w x=k \mu \hat{\mu}$ where $\mu$ and $\hat{\mu}$ are paths and $e$ is an exit for $c$ at $r(\mu)$. Then we have

$$
e^{*} \mu^{*} x=e^{*} \mu^{*} w x=k e^{*} \mu^{*} \mu \hat{\mu}=k e^{*} \hat{\mu}=0 .
$$

Now $\mu e$ is a path starting in $\Theta_{0}$ and thus $r(e) \in \Theta_{0}=\mathcal{V} \cup \mathcal{P} \cup \mathcal{G}$. If $r(e) \in \mathcal{V}$ then

$$
x e^{*} \mu^{*}=x r(e) e^{*} \mu^{*}=r(e) x e^{*} \mu^{*}=k r(e) e^{*} \mu^{*} \neq 0,
$$

which contradicts the fact that $x \in Z$. Similarly, if $r(e) \in \mathcal{P}$ then there is a closed path $\hat{c}$ so that $r(e) x=k \hat{c}$ and hence

$$
x e^{*} \mu^{*}=x r(e) e^{*} \mu^{*}=k \hat{c} e^{*} \mu^{*} \neq 0 .
$$

If $r(e) \in \mathcal{G}$ then there exists a closed path $\hat{d}$ so that $r(e) x=k \hat{d}^{*}$ and thus

$$
x e^{*} \mu^{*}=x r(e) e^{*} \mu^{*}=k \hat{d}^{*} e^{*} \mu^{*} \neq 0 .
$$

In each case we get a contradiction and hence we must have that $\mathcal{P}=\emptyset$. Following analogous steps, we obtain that $\mathcal{G}$ is also empty. Thus we have that $w x=k w$ for every $w \in \Theta_{0}$.

Next we will use induction to show that every vertex $w$ in $E^{0}$ has the property that $w x=k w$. For every $n \geq 1$, let

$$
\Theta_{n}=\left\{z \in E^{0} \mid \emptyset \neq r\left(s^{-1}(z)\right) \subseteq \Theta_{n-1}\right\} \cup \Theta_{n-1}
$$

and suppose that $w x=k w$ for every $w \in \Theta_{n-1}$.

If $z \in \Theta_{n} \backslash \Theta_{n-1}$ then

$$
z=\sum_{e \in s^{-1}(z)} e e^{*}=\sum_{e \in s^{-1}(z)} e r(e) e^{*}
$$

and hence

$$
z x=\sum_{e \in s^{-1}(z)} e r(e) x e^{*}=\sum_{e \in s^{-1}(z)} k e r(e) e^{*}=k z .
$$

Since $\Theta_{0}$ has the property that $z x=k z$ for every $z \in \Theta_{0}$, we have shown that given any integer $n$, we have $z x=k z$ for every $z \in \Theta_{n}$.

Since, as noted in Section 1, $\bigcup_{n=0}^{\infty} \Theta_{n}=\overline{\{(r(p))\}} \neq \emptyset$ and $E^{0}$ has no proper hereditary and saturated subsets, we have that $E^{0}=\bigcup_{n=0}^{\infty} \Theta_{n}$ and thus every vertex $z$ of $E$ has the property that $z x=k z$. 
Therefore we have

$$
x=\sum_{v \in E^{0}} v x=\sum_{v \in E^{0}} k v=k \sum_{v \in E^{0}} v .
$$

Now if $E^{0}$ is finite we have that

$$
x=\sum_{v \in E^{0}} v x=\sum_{v \in E^{0}} k v=k \cdot 1 .
$$

Thus $Z \subseteq K \cdot 1_{L(E)}$. Since elements of $K$ commute with elements of $L(E)$, we obtain that $Z=K \cdot 1_{L(E)}$.

On the other hand, if $E^{0}$ is infinite then there is a finite, nonempty collection of vertices $\mathcal{C}$ so that

$$
x=\sum_{v \in \mathcal{C}} v x=\sum_{v \in \mathcal{C}} k v .
$$

Since $E^{0}$ is infinite, there is a vertex $v^{\prime} \notin \mathcal{C}$, and hence we have

$$
x=\sum_{x \in \mathcal{C}} k v+0 \cdot v^{\prime} .
$$

Note that the set of vertices in each connected component of $E$ is a nonempty hereditary and saturated subset of $E^{0}$ and hence $E^{0}$ must be the only such set. Thus $E$ is connected so we may apply Lemma 4.1 and obtain that $k$ must equal 0 and hence $x=0$. Therefore in the case that $E^{0}$ is infinite we obtain that $Z=0$.

In his seminal papers $[21,22]$ Leavitt studied the classical Leavitt algebras $L(1, n)$ for $n \geq 2$. It is known that these are simple (in fact purely infinite simple). Here we show that they are central.

Corollary 4.3. The Leavitt algebra $L(1, n)$ is a central simple algebra for $n \geq 2$.

Proof. We already noted that $L(1, n)$ can be realized as the Leavitt path algebra of the rose with $n$ petals graph $R_{n}$. Since $L(1, n)$ is simple and unital, Theorem 4.2 gives that $Z(L(1, n))=K$.

Using almost disjoint unions we can compute the center of Leavitt path algebras whose subgraphs give simple Leavitt path algebras.

Corollary 4.4. If $E=\coprod_{i \in I} E_{i}$, where $L\left(E_{i}\right)$ is simple for each $i \in I$, then $Z(L(E)) \cong \bigoplus_{i \in J} K$, where $J=\left\{i \in I \mid E_{i}\right.$ is finite $\}$.

Proof. Apply Proposition 2.4 together with Theorem 4.2 and the fact that $L\left(E_{i}\right)$ is unital if and only if $E_{i}$ is finite. 


\section{The center of exchange Leavitt path algebras}

It is well-known that simple Leavitt path algebras are all exchange $[2$, Lemma $7]$ and [12, Theorem 4.5]. This is the algebraic translation of the fact that Condition (L) plus cofinality implies Condition (K) for graphs. Thus, paralleling what happens in the graph $C^{*}$-algebra literature for $C^{*}(E)$, Condition $(\mathrm{K})$ is the next natural framework to work in once the phenomena under Condition (L) and cofinality are understood for $L(E)$.

The study of the center of Leavitt path algebras will be no exception to this rule. Thus, in this section we explore some generalizations of Theorem 4.2: concretely, we compute the center of the exchange Leavitt path algebras which satisfy certain weak finiteness conditions.

While the next lemma is known, we could not find a reference in the literature. We provide a proof here for completeness.

Lemma 5.1. Let $A$ be a $K$-subalgebra of $\bigoplus_{i=1}^{n} K$ where $n \geq 0$. Then $A$ is isomorphic as a $K$-algebra to $\bigoplus_{i=1}^{m} K$ for some $m \in\{0, \ldots, n\}$ (where for $m=0$ the sum reduces to $\{0\})$.

Proof. Suppose the result holds for $n-1$ and we will prove it for $n$. Let $d=$ $\operatorname{dim}_{K}(A)$. Assume $n \geq 2$ and $d<n$ (the other cases are trivial), then there exist coefficients $a_{1}, \ldots, a_{n} \in K$, not all zero, such that $a_{1} k_{1}+\cdots+a_{n} k_{n}=0$ for every $\left(k_{1}, \ldots, k_{n}\right)$ in $A$. Suppose that $a_{i} \neq 0$ so that the equation

$$
k_{i}=-\frac{a_{1}}{a_{i}} k_{1}-\cdots-\frac{a_{i-1}}{a_{i}} k_{i-1}-\frac{a_{i+1}}{a_{i}} k_{i+1}-\cdots-\frac{a_{n}}{a_{i}} k_{n}
$$

is satisfied for every $\left(k_{1}, \ldots, k_{n}\right) \in A$ as well. This implies that $\varphi: A \rightarrow$ $\bigoplus_{i=1}^{n-1} K$ given by $\varphi\left(k_{1}, \ldots, k_{n}\right)=\left(k_{1}, \ldots, k_{i-1}, k_{i+1}, \ldots, k_{n}\right)$ is $K$-algebra monomorphism so the induction hypothesis applies.

Recall that a ring $R$ has a two-sided composition series of length $n$ if there exists an ascending chain of two-sided ideals of $R$ :

$$
0=I_{0} \subseteq I_{1} \subseteq \cdots \subseteq I_{n}=R
$$

such that, for every $0 \leq i \leq n-1, I_{i}$ is maximal among the ideals of $R$ contained in $I_{i+1}$.

As usual, let us denote by $\mathcal{L}(L(E))$ (resp. $\left.\mathcal{L}_{\text {gr }}(L(E))\right)$ the set of all ideals (resp. graded ideals) of $L(E)$.

Proposition 5.2. If $L(E)$ is an exchange Leavitt path algebra with a twosided composition series of finite length $n$, then there exists a $K$-algebra isomorphism $Z(L(E)) \cong \bigoplus_{i=1}^{m} K$ for some $m \in\{0, \ldots, n\}$. 
Proof. Let us consider an ascending chain of two-sided ideals

$$
0=I_{0} \subseteq I_{1} \subseteq \cdots \subseteq I_{n}=L(E)
$$

with $n \geq 1$ such that, for every $0 \leq i \leq n-1, I_{i}$ is maximal among the ideals of $L(E)$ contained in $I_{i+1}$. We will prove the result by induction on $n$.

If $n=1$, then $L(E)$ is indeed a simple algebra so that, by Theorem 4.2, we obtain that $Z(L(E))=K \cdot 1_{L(E)}$ in case that $L(E)$ is unital or $Z(L(E))=0$ otherwise. In both situations the conclusion of the Proposition is obviously satisfied by taking $m=1$ or $m=0$ respectively.

Suppose now that the result holds for $k<n$. By [12, Theorem 4.5] and [8, Theorem 4.3], there exist hereditary and saturated sets $H_{i}(0 \leq i \leq n)$ such that

(i) $I_{i}=I\left(H_{i}\right)$ for every $0 \leq i \leq n$; in particular, $H_{i} \varsubsetneqq H_{i+1}$ for every $0 \leq i \leq n-1$, and

(ii) for any $0 \leq i \leq n-1$, there does not exist a hereditary and saturated set $T$ such that $H_{i} \varsubsetneqq T \varsubsetneqq H_{i+1}$.

The quotient graph $E / H_{1}$ inherits Condition (K) from $E$ by [12, Lemma 3.2]. Thus, another application of [12, Theorem 4.5$]$ yields that $\mathcal{L}_{\mathrm{gr}}\left(L\left(E / H_{1}\right)\right)=$ $\mathcal{L}\left(L\left(E / H_{1}\right)\right)$.

For each $1 \leq i \leq n$, let $J_{i} \triangleleft L\left(E / H_{1}\right)$ be the ideal generated by $H_{i}$ inside $L\left(E / H_{1}\right)$. Then the previous remarks imply that

$$
0=J_{1} \subseteq J_{2} \subseteq \cdots \subseteq J_{n}=L\left(E / H_{1}\right)
$$

is a chain of two-sided ideals of $L\left(E / H_{1}\right)$ such that for every $1 \leq i \leq n-1$, $J_{i}$ is maximal among the ideals of $L\left(E / H_{1}\right)$ contained in $J_{i+1}$. Otherwise, since $\mathcal{L}_{\text {gr }}\left(L\left(E / H_{1}\right)\right)=\mathcal{L}\left(L\left(E / H_{1}\right)\right)$, [12, Lemma 2.5] would contradict property (ii) satisfied by the sets $H_{i}$. In this situation, the induction hypothesis gives that $Z\left(L\left(E / H_{1}\right)\right) \cong \bigoplus_{i=1}^{t} K$ for some $t \geq 0$.

Let $I:=I_{1}=I\left(H_{1}\right)$ and note that, because $I$ is a graded ideal, an application of $[10$, Lemma 1.2$]$ implies that $I \cong L\left(H_{1} E\right)$. Moreover, $I$ is simple as a $K$-algebra because under Condition $(\mathrm{K})$ any ideal of $I$ would also be an ideal of $L(E)$ (see [12, Lemma $5.3(1)]$ ). Hence, we can apply our main result, Theorem 4.2, to the Leavitt path algebra $I \cong L\left({ }_{H_{1}} E\right)$, so that we have two cases depending on whether or not the ideal $I$ has a unit.

First, if $I$ has a unit, say $1_{I} \in I$, then Theorem 4.2 gives $Z(I)=K \cdot 1_{I}$, and we proceed as follows. Let $J:=\left\{x-x 1_{I} \mid x \in L(E)\right\}$, which is clearly a left ideal of $L(E)$. Note that $1_{I}$ is a central idempotent in $L(E)$ because if $z \in L(E)$ is arbitrary, then the facts that $I$ is a two-sided ideal and that $1_{I} \in Z(I)$ yield

$$
z 1_{I}=\left(z 1_{I}\right) 1_{I}=1_{I}\left(z 1_{I}\right)=\left(1_{I} z\right) 1_{I}=1_{I}\left(1_{I} z\right)=1_{I} z .
$$


Therefore $J$ coincides with the set $\left\{x-1_{I} x \mid x \in L(E)\right\}$ and hence is a right ideal as well. It is obvious that $L(E)=I+J$, and the sum is direct because if $y \in I$ and $y=x-x 1_{I}$ for some $x \in L(E)$ then $x \in I$ so $y=x-x 1_{I}=x-x=0$. This finally gives an algebra isomorphism $L(E) / I \cong J$ so that in particular $L(E) \cong I \oplus L(E) / I$ and then

$$
Z(L(E)) \cong Z(I) \oplus Z\left(L(E) / I\left(H_{1}\right)\right) \cong Z(I) \oplus Z\left(L\left(E / H_{1}\right)\right)
$$

where the last isomorphism is given by [12, Lemma $2.3(1)]$. Now since $Z\left(L\left(E / H_{1}\right)\right) \cong \bigoplus_{i=1}^{t} K$ for some $t \geq 0$ by hypothesis, $Z(L(E)) \cong \bigoplus_{i=1}^{t+1} K$ for some $t \geq 0$ and this case is completed.

For the second case, assume that $I$ does not have a unit. Then Theorem 4.2 gives $Z(I)=0$. Consider the $K$-algebra epimorphism $\pi: L(E) \rightarrow$ $L(E) / I$. Since clearly $\pi(Z(L(E))) \subseteq Z(L(E) / I)$ we also have a $K$-algebra homomorphism $\left.\pi\right|_{Z(L(E))}: Z(L(E)) \rightarrow Z(L(E) / I)$ which in turn is injective by the following argument: if $x \in Z(L(E))$ is such that $\bar{x}=\overline{0}$ in $Z(L(E) / I)$ then $x \in I \cap Z(L(E)) \subseteq Z(I)=0$. This shows that $Z(L(E)) \cong \operatorname{Im}\left(\left.\pi\right|_{Z(L(E))}\right)$ as $K$-algebras. But since $\operatorname{Im}\left(\left.\pi\right|_{Z(L(E))}\right)$ is a $K$ subalgebra of $Z(L(E / I)) \cong \bigoplus_{i=1}^{t} K$ for some $t \geq 0$, then Lemma 5.1 applies to obtain the desired conclusion.

Example 5.3. The variable $m$ in Proposition 5.2 may indeed take any value within the range $\{0, \ldots, n\}$. That is, given any $n>0$ there exists a family of graphs $\left\{E_{0}, \ldots, E_{n}\right\}$ so that for any $0 \leq m \leq n$, the algebra $L\left(E_{m}\right)$ has a 2-sided composition series of length $n$ and $Z\left(L\left(E_{m}\right)\right) \cong \bigoplus_{i=1}^{m} K$.

To exhibit a family of examples with this behavior we consider the most basic examples of both unital and nonunital simple Leavitt path algebras. Thus, let $F$ be the graph consisting on one isolated vertex only and let $G$ be the infinite line graph, as in the graph exhibited below.

$$
F=\bullet \quad G=\bullet \longrightarrow \bullet \longrightarrow \bullet \longrightarrow \cdots \cdots \ldots \ldots
$$

It is well-known that $L(F) \cong K$ and that $L(G) \cong \mathbb{M}_{\infty}(K)$. Let $m \in$ $\{0, \ldots, n\}$. For each $i=1, \ldots, m$ let $F_{i}$ be a copy of $F$ and for each $i=$ $m+1, \ldots, n$ let $G_{i}$ be a copy of $G$. Define $E_{m}$ as the disjoint union of these graphs; that is $E_{m}=\left(\coprod_{i=1}^{m} F_{i}\right) \coprod\left(\coprod_{i=m+1}^{n} G_{i}\right)$. It is straightforward to check that

$$
\emptyset \subseteq F_{1}^{0} \subseteq F_{1}^{0} \cup F_{2}^{0} \subseteq \cdots \subseteq \bigcup_{i=1}^{m} F_{i}^{0} \cup G_{m+1}^{0} \subseteq \cdots \subseteq \bigcup_{i=1}^{m} F_{i}^{0} \cup \bigcup_{i=m+1}^{n} G_{i}^{0}=E_{m}^{0}
$$

is a chain of hereditary and saturated subsets of $E_{m}^{0}$ that cannot be refined (that is, there does not exist $H \in \mathcal{H}_{E_{m}}$ strictly contained between two consecutive elements of the chain).

Since $E_{m}$ is acyclic, it satisfies Condition $(K)$ and therefore, as noted previously, the two-sided ideals of $E_{m}$ correspond to hereditary and satura- 
ted subsets of vertices of $E_{m}$ and this readily implies that the chain of ideals

$$
\begin{aligned}
0 & =I(\emptyset) \subseteq I\left(F_{1}^{0}\right) \subseteq \cdots \subseteq I\left(\bigcup_{i=1}^{m} F_{i}^{0} \cup G_{m+1}^{0}\right) \subseteq \cdots \\
& \subseteq I\left(\bigcup_{i=1}^{m} F_{i}^{0} \cup \bigcup_{i=m+1}^{n} G_{i}^{0}\right)=L\left(E_{m}\right)
\end{aligned}
$$

is a two-sided composition series of length $n$ for $L\left(E_{m}\right)$. Finally, an application of Proposition 2.4 gives that $L\left(E_{m}\right)=\bigoplus_{i=1}^{m} L\left(F_{i}\right) \oplus \bigoplus_{i=m+1}^{n} L\left(G_{i}\right)$, so in particular $Z\left(L\left(E_{m}\right)\right) \cong \bigoplus_{i=1}^{m} K$.

Remark 5.4. Furthermore, Remark 5.3 might suggest that the number $m$ in Proposition 5.2 is precisely the number of (simple) quotients $I_{i+1} / I_{i}$ which happen to have nonzero center (therefore isomorphic to $K$ ) in the proof of that proposition. The following example shows that this might not be the case. If $E$ is the graph

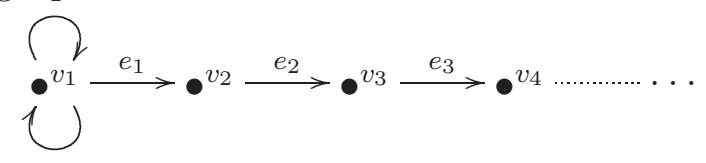

then $H=\left\{v_{2}, v_{3}, \ldots\right\}$ is the only nontrivial hereditary and saturated subset of vertices and since $E$ satisfies Condition $(\mathrm{K})$, the series $0 \subseteq I=I(H) \subseteq$ $L(E)$ is a two-sided composition series of length 2 for $L(E)$. We will show that $Z(L(E) / I)=K$ and $Z(I)=0$, yet we have $Z(L(E))=0 \neq K \oplus 0$.

Indeed, following the proof of Proposition 5.2 we obtain that $\left.I \cong{ }_{H} E\right)$ where the graph ${ }_{H} E$ is given by

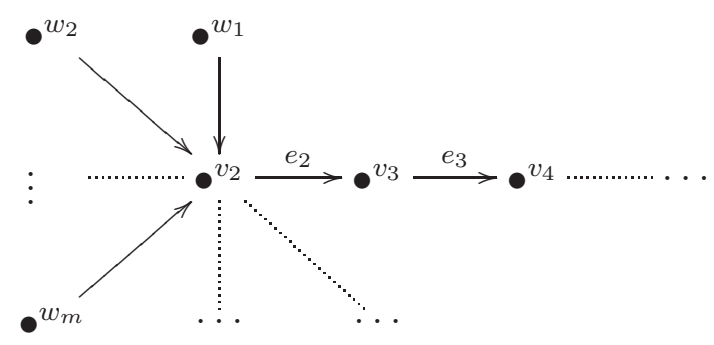

and there are infinitely many vertices $w_{i}$ pointing to $v_{2}$. Because this graph yields a simple algebra by [1, Theorem 3.11] (and nonunital, as it has an infinite number of vertices), then $Z(I)=0$ by Theorem 4.2 . In this situation we had a $K$-algebra monomorphism $\left.\pi\right|_{Z(L(E))}: Z(L(E)) \hookrightarrow Z(L(E) / I)$. But now, since $E / H$ is the rose with two petals graph

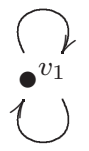


we have that $L(E / H) \cong L(1,2)$ is simple (and unital) so Theorem 4.2 gives that $Z(L(E / H)) \cong K$. Of course $L(E / H) \cong L(E) / I(H)=L(E) / I$, so $Z(L(E) / I) \cong K$.

Suppose that $0 \neq x \in Z(L(E))$. Since the finite sums of vertices form a set of local units for $L(E)$, then the "line" structure of $E$ guarantees the existence of $i \in \mathbb{N}$ such that $x v_{i} \neq 0=x v_{i+1}, e_{i} e_{i}^{*}=v_{i}$ and $r\left(e_{i}\right)=v_{i+1}$. Putting these things together we get

$$
0 \neq x v_{i}=x\left(e_{i} e_{i}^{*}\right)=\left(e_{i} x\right)\left(s\left(e_{i}^{*}\right) e_{i}^{*}\right)=\left(e_{i} x\right)\left(r\left(e_{i}\right) e_{i}^{*}\right)=e_{i}\left(x v_{i+1}\right) e_{i}^{*}=0 .
$$

Since we have a contradiction, we must have that $Z(L(E))=0$. Note that the reason why $Z(L(E)) \approx Z(I) \oplus Z(L(E) / I)$ is that the monomorphism $\left.\pi\right|_{Z(L(E))}: Z(L(E)) \rightarrow Z(L(E) / I)$ is not an epimorphism.

The following corollaries are particular and frequent cases of Proposition 5.2.

Corollary 5.5. Let $L(E)$ be an exchange Leavitt path algebra such that $|\mathcal{L}(L(E))|=l<\infty$. Then there exists a $K$-algebra isomorphism $Z(L(E)) \cong$ $\bigoplus_{i=1}^{m} K$ for some $m \in\{0, \ldots, l\}$.

Proof. Clearly $L(E)$ has a two-sided composition series of length $n \leq l$ so that Proposition 5.2 applies.

Corollary 5.6. If $L(E)$ is a unital exchange Leavitt path algebra, then there exists a $K$-algebra isomorphism $Z(L(E)) \cong \bigoplus_{i=1}^{m} K$ for some $m \geq 1$.

Proof. Since $L(E)$ is unital, $E^{0}$ is finite and thus so is the set of hereditary and saturated sets of vertices $\mathcal{H}_{E}$, which is isomorphic as a lattice to the set $\mathcal{L}_{\text {gr }}(L(E))$ of all graded left ideals of $L(E)$ by [8, Theorem 4.3]. Furthermore, by [12, Theorem 4.5], the fact that $L(E)$ is exchange gives that $\mathcal{L}_{\text {gr }}(L(E))=\mathcal{L}(L(E))$. Corollary 5.5 gives us $m \geq 0$ with the desired property and note that $m=0$ is not possible as $1 \in Z(L(E))$.

Remark 5.7. It is worth pointing out that $L(E)$ being exchange such that $|\mathcal{L}(L(E))|=l<\infty$ does not imply that $L(E)$ is unital (the graph $E$ in Remark 5.4 is a counterexample). In other words, Corollary 5.5 really is more general than Corollary 5.6.

Acknowledgments. Part of this work was carried out during visits of the first author to the University of Calgary and of the second author to the Universidad de Málaga. They thank these host centers for their warm hospitality and support. 


\section{References}

[1] Abrams, G. And Aranda Pino, G.: The Leavitt path algebra of a graph. J. Algebra 293 (2005), no. 2, 319-334.

[2] Abrams, G. and Aranda Pino, G.: Purely infinite simple Leavitt path algebras. J. Pure Appl. Algebra 207 (2006), no. 3, 553-563.

[3] Abrams, G. and Aranda Pino, G.: The Leavitt path algebras of arbitrary graphs. Houston J. Math. 34 (2008), no. 2, 423-442.

[4] Abrams, G., Aranda Pino, G. and Siles Molina, M.: Finitedimensional Leavitt path algebras. J. Pure Appl. Algebra 209 (2007), no. 3, $753-762$.

[5] Abrams, G., Aranda Pino, G. and Siles Molina, M.: Locally finite Leavitt path algebras. Israel J. Math. 165 (2008), 329-348.

[6] Abrams, G., Aranda Pino, G., Perera, F. and Siles Molina, M.: Chain conditions for Leavitt path algebras. Forum Math. 22 (2010), 95-114.

[7] Abrams, G. and Tomforde, M.: Isomorphism and Morita equivalence of graph algebras. Trans. Amer. Math. Soc. 363 (2011), no. 7, 3733-3767.

[8] Ara, P., Moreno, M. A. And Pardo, E.: Nonstable $K$-theory for graph algebras. Algebr. Represent. Theory 10 (2007), 157-178.

[9] Ara, P. and Mathieu, M.: Local multipliers of $C^{*}$-algebras. Springer Monographs in Mathematics. Springer-Verlag London, London, 2003.

[10] Ara, P. And Pardo, E.: Stable rank of Leavitt path algebras. Proc. Amer. Math. Soc. 136 (2008), no. 7, 2375-2386.

[11] Aranda Pino, G., Martín Barquero, D., Martín González, C. And Siles Molina, M.: The socle of a Leavitt path algebra. J. Pure Appl. Algebra 212 (2008), no. 3, 500-509.

[12] Aranda Pino, G., Pardo, E. and Siles Molina, M.: Exchange Leavitt path algebras and stable rank. J. Algebra 305 (2006), no. 2, 912-936.

[13] Aranda Pino, G., Pardo, E. and Siles Molina, M.: Prime spectrum and primitive Leavitt path algebras. Indiana Univ. Math. J. 58 (2009), no. 2, 869-890.

[14] Aranda Pino, G., Perera, F., and Siles Molina, M. (eds.): Graph algebras: bridging the gap between analysis and algebra. University of Málaga Press, Málaga, Spain, 2007.

[15] Bates, T., Hong, J. H., Raeburn, I. and Szymański, W.: The ideal structure of the $C^{*}$-algebras of infinite graphs. Illinois J. Math. 46 (2002), no. $4,1159-1176$.

[16] Deicke, K., Hong, J.H. And Szymański, W.: Stable rank of graph algebras. Type I graph algebras and their limits. Indiana Univ. Math. J. 52 (2003), no. 4, 963-979.

[17] Firkins, J.: Locally artinian serial rings. Comm. Alg. 32 (2004), no. 4, $1255-1264$. 
[18] Fuller, K.: On rings whose left modules are direct sums of finitely generated modules. Proc. Amer. Math. Soc. 54 (1976), 39-44.

[19] Goodearl, K. R.: Leavitt path algebras and direct limits. In Rings, modules and representations, 165-187. Contemp. Math. 480. Amer. Math. Soc., Providence, RI, 2009.

[20] Kumjian, A., Pask, D., Raeburn, I. and Renault, J.: Graphs, groupoids and Cuntz-Krieger algebras. J. Funct. Anal. 144 (1997), 505-541.

[21] Leavitt, W. G.: The module type of a ring. Trans. Amer. Math. Soc. 103 (1962), 113-130.

[22] Leavitt, W. G.: The module type of homomorphic images. Duke Math. J. 32 (1965), 305-311.

[23] Raeburn, I.: Graph algebras. CBMS Regional Conference Series in Mathematics 103. Amer. Math. Soc., Providence, RI, 2005.

[24] Siles Molina, M.: Algebras of quotients of path algebras. J. Algebra 319 (2008), no. $12,5265-5278$.

[25] Tomforde, M.: Uniqueness theorems and ideal structure for Leavitt path algebras. J. Algebra 318 (2007), no. 1, 270-299.

Recibido: 23 de febrero de 2009

Gonzalo Aranda Pino

Departamento de Álgebra, Geometría y Topología Universidad de Málaga 29071, Málaga, Spain

g. aranda@uma.es

Kathi Crow Department of Mathematics

Salem State University Salem MA 01970, USA kcrow@salemstate.edu

The first author was partially supported by a Postdoctoral Fellowship by the MEC and FECYT agency through grant EX2007-1069; by the Spanish MEC and Fondos FEDER jointly through projects MTM2007-60333 and MTM2010-15223; by PAI III projects FQM-336, FQM-1215 and FQM-02467 of the Junta de Andalucía; and by the Consolider Ingenio "Mathematica" project CSD2006-32 by the MEC. The second author was partially supported by a "Short stays of foreign researchers" program by the Universidad de Málaga. 